\title{
EFFORTS LONGITUDINAUX PROVOQUÉS DANS LES CONDUITES FORCÉES PAR LA PRESSION HYDRAULIQUE \& LES VARIATIONS DE TEMPÉRATURE
}

\author{
H. GERODOLLE \\ Ingénieur A. \& $M$.
}

Les efforts longitudinaux, sur les conduies forcées, ancrées à deux extrémités dans des massifs, peurent prendre une valeur importante, non seulement par les effets dus à la température mais aussi par la contraction ou dilatation longitudinale, provoquée par la pression intérieure.

Les effets de la pression sont tres souvent négligés alors que le calcul en est très simple, même pour des conduites formées de tronçons différents.

Nous nous proposons, dans ce qui suil, d'étudier les cas les plus généraux en tenant compte de tous les effets, thermiques et hydrauliques.

Les notations que nous allons employer, par la suite, sont les suivantes :

$\mathrm{L}=$ longueur de la conduite

$\mathrm{D}=$ diamètre intérieur de la conduite

$e=$ épaisseur de la conduite

$\mathrm{S}=\frac{\pi \mathrm{D}^{2}}{4}=$ section intérieure de la conduite

$\Omega=\pi \mathrm{D} e=$ section de métal de la conduite

$p=$ pression hydraulique en un point donnc par unité de surface

$\mathrm{Q}=p \frac{\pi \mathrm{D}^{2}}{4}=$ effort total qui s'exercerait sur une section déterminée de la conduile si elle était obturée.

$\mathrm{E}=$ module d'élasticité du métal

$\mathrm{n}=$ coefficient de Poisson : 0,3 pour l'acier ; 0,25 pour la fonte

$\alpha=$ coefficient de dilatation linéaire due à la température

$t_{0}=$ température de pose de la conduite

$t=$ température du métal de la conduite

$\mathrm{B}=$ dilatation ou contraction longitudinale de la conduite due à la température $\mathrm{B}=b 1+b 2+\ldots ; b 1, b 2$, etc., étant les dilatations ou contractions des éléments différents constituant la conduite $\mathrm{BO}=$ dilatation $; \mathrm{BO}=$ contraction.

$C=$ dilatation ou contraction longitudinale de la conduite due à la pression intérieure, $\mathrm{C}=c 1+c 2+\ldots ; c 1, c 2$, etc. étant les dilatations ou contractions des éléments différents constituant la conduite, $\quad \mathrm{C}>\mathrm{O}=$ dilatation, $\mathrm{C}<\mathrm{O}$ $=$ contraction.

$\Delta=B+C$ déplacement total

$K=\frac{1}{E \Omega}=$ flexibilité longitudinale totale d'une conduite formée de plusieurs éléments dont la flexibilité propre est $k 1, k 2, k 3$ etc... K $=k 1+k 2+k 3+$ ete.

$F=$ effort de traction ou de compression longitudinale en un point de la conduite, $\mathrm{F}-\mathrm{O}=$ traction, $\mathrm{F}<\mathrm{O}=$ compression.

Nous allons commencer par indiquer les formules pour les cas simples, c'est-à-dire pour des conduites à caractéristiques uniformes.

Enonçons d'abord, la règle générale suivante :

- Les déformations ou les efforts longitudinaux dus aux effets de la pression hydraulique s'ajoutent algébriquement aux déformations ou aux efforts longitudinaux dus anx variations de température.

On peut donc calculer séparément ces efforts ou ces déformations et les ajouter algébriquement à la fin du calcul.

\section{I. - EFFETS DES VARIATIONS DE TEMPE- RATURE.}

a) Troncon de condaite à déplacement libre. Sur les conduites à déplacement libre, l'effort dù aux variations de température est nul. 

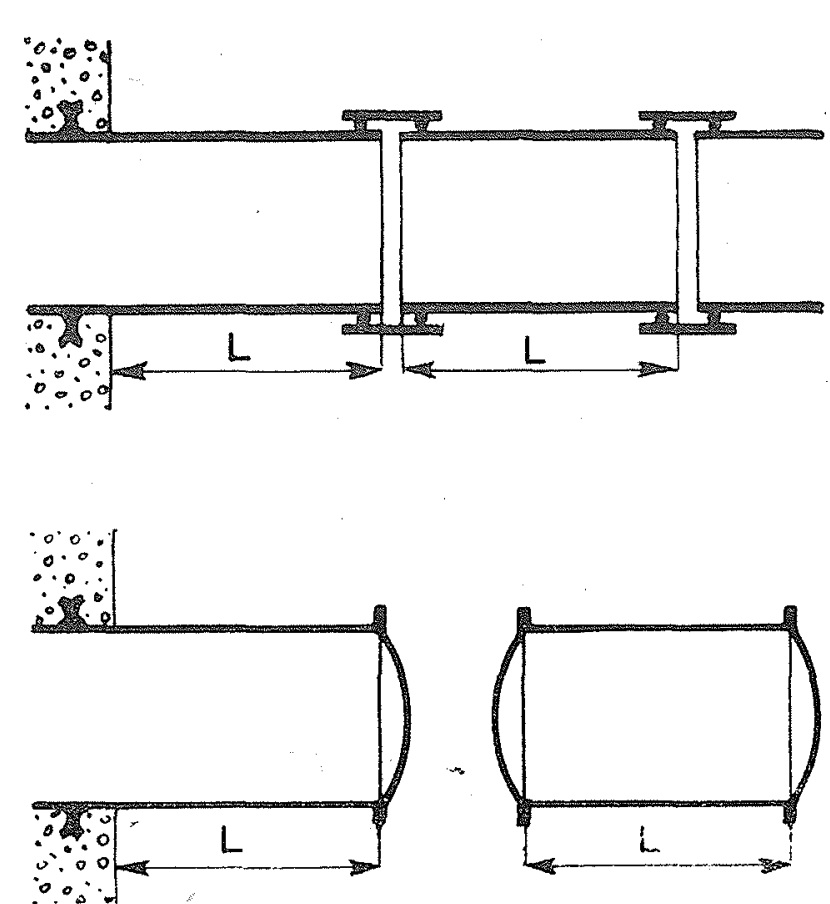

Fig. 1

$\mathrm{F}=0$

déplacement $b=\left(t-t_{0}\right) \propto 1$.

Le coefficient de dilatation $\alpha=0,0000122$ pour l'acier, et 0,000010 pour la fonte.

II est plus commode de se rappeler que la dilatation on la contraction est :

Pour une variation de $100^{\circ}$

$1,2 \mathrm{~m} / \mathrm{m}$ par mètre pour l'acier,

$1 \mathrm{~m} / \mathrm{m}$ par mètre pour la fonte.

b) Tronȩon de conduite à déplacement impossible.
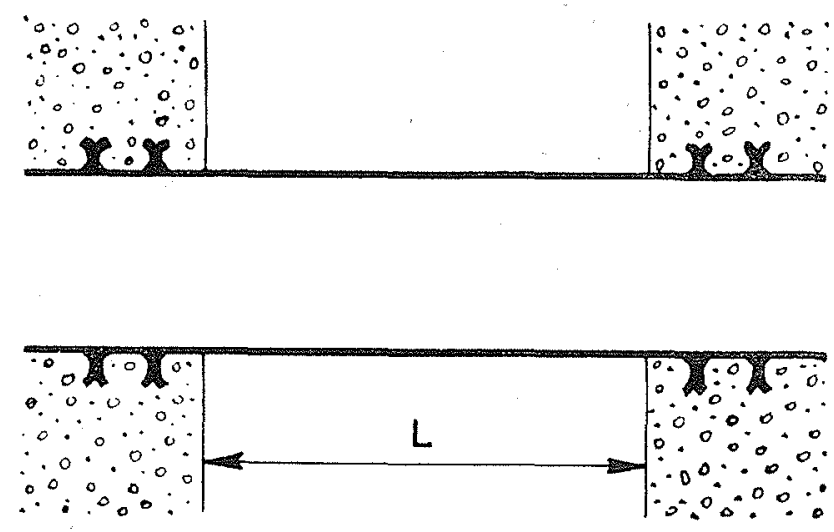

Fig. 2

Pour qu'il en soit ainsi, il faul que les deux extrémités de la conduite soient encastrées dans deux massifs en maconnerie supposés fixes.
Les dilatations ou contractions étant impossibles, il s'ensuit un effort dans la conduite :

-.... de traction, s'il y a baisse de température par rapport à la température de pose ;

- de compression, au contraire, s'il y a élévation de température.

$b=0 ; \quad \mathrm{F}=-\alpha\left(t-t_{1}\right) \mathrm{E} \Omega$, si $t-t_{11}<$ 0 refroidissement, $i>0$ traction.

si $t-t_{1}>0$ echauitement, $F<0$ compression.

La contrainte de métal est :

$$
\sigma=-\alpha\left(l-t_{u}\right) \mathrm{E}
$$

Mais il est plus commode, si l'on cherche la contrainte, d'employer les formules pratiques suivantes :

$$
\sigma=-\frac{t-t_{11}}{1} \mathrm{~kg} / \mathrm{mm}^{2} \text { pour l'acier avec }
$$

$\mathrm{E}=20$ a $21000 \mathrm{~kg} / \mathrm{mm}^{2}$

$$
\sigma=-\frac{1-t_{i s}}{10} \mathrm{~kg} / \mathrm{mm}^{2} \text { pour in fonte avec }
$$

$\mathrm{E}=10000 \mathrm{~kg} / \mathrm{mm}^{2}$

Par exemple: Une augmentation de température de $30^{\circ}$ par rapport à la température de pose, donnera une contrainte de :

$$
\sigma=-\frac{30}{4}=-7,5 \mathrm{~kg} / \mathrm{mm}^{2} \text {. }
$$

o étant négatif, est donc une compression.

II est à remarquer que :

- Ia longueur de la conduite n'intervient pas;

- la contrainte dépend uniquement de la température ;

- l'effort total sur la conduite et, par conséquent, sur les massifs d'ancrage, est proportionnel a $\Omega$ section de métal de la conduite.

L'effort total sur la conduite peut s'écrire :

$$
\mathrm{F}=\frac{b}{k}
$$

i étant le déplacemenl de la conduite supposée libre de se déplacer.

$$
b=\left(t-t_{0}\right) \times l
$$

i. étant la flexibilité longitudinale de la conduite $r=\frac{l}{\mathrm{E} \Omega}$

\section{II. - EFFETS DUS A LA PRESSION HYDRAU. LIQUE}

a) Troncon de conduite avec joints coulissants :

$\begin{aligned} \mathrm{F} & =0 \\ \text { déplacement } \quad c & =-\mathrm{n} \frac{p \mathrm{D}}{2 e} \quad \frac{\mathrm{L}}{\mathrm{E}} \text { c'est une }\end{aligned}$ contraction. 


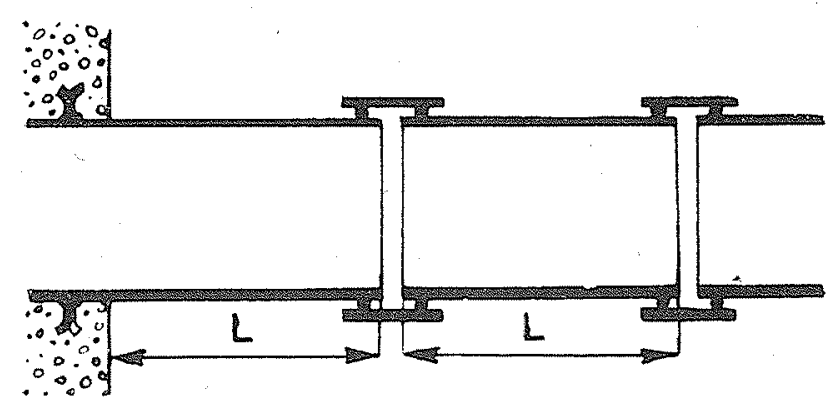

Fig. 3

On peut écrire : $c=-2$ ก $Q$ k $\mathrm{car}=\frac{1}{\mathrm{E} \Omega}, Q=p \frac{\pi \mathrm{D} 2}{4}$, et $\Omega=\pi \mathrm{De}$

b) Troncon de conduite a deplacement libre el muni d'un obtaraten :
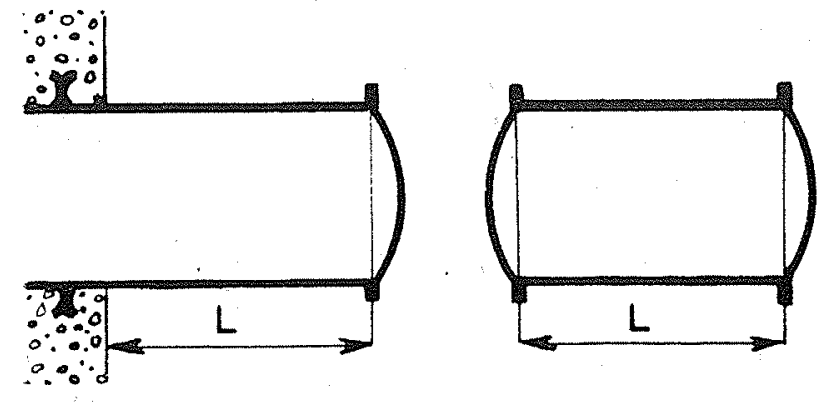

Fig. 4

$$
\begin{aligned}
\mathrm{F}= & \mathrm{Q}=p \frac{\pi \mathrm{D}}{4} \\
\text { Aeplacement } c= & (1 / 2-\mathrm{n}) \frac{p \mathrm{D}}{2 e} \frac{\mathrm{L}}{\mathrm{E}} \text { dila- } \\
& \text { tation car } \mathrm{n}<1 / 2 \\
\text { ou } c= & (1 / 2-\mathrm{n}) \mathrm{Q} k .
\end{aligned}
$$

c) Troncon de conduite à déplacement longitudinal impossible :
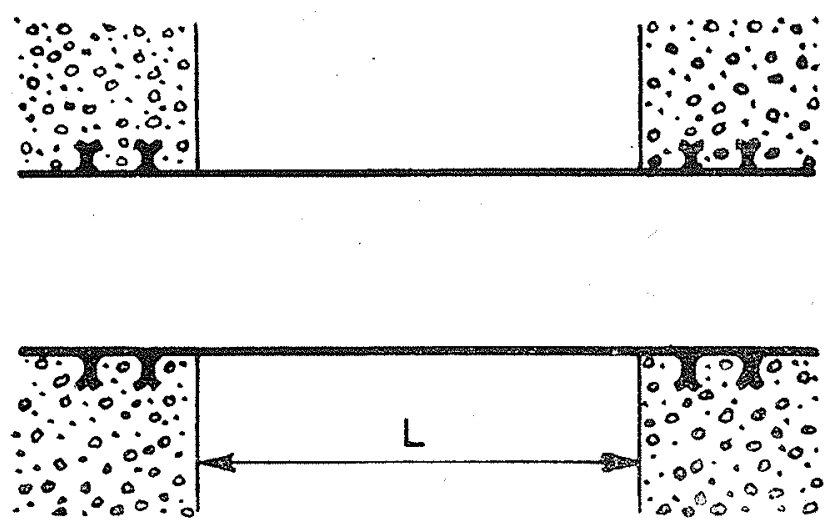

Fig. 5

$$
\begin{aligned}
c & =0 \text { déplacement nul. } \\
\mathrm{F} & =\mathrm{n} \frac{p \mathrm{D}}{2 e} \Omega \text { traction } \\
\text { ou } \mathrm{F} & =2 \mathrm{n} \mathrm{Q} \quad \mathrm{Q}=p \frac{\pi \mathrm{D}}{4}
\end{aligned}
$$

\section{CAS GENERAUX}

A l'aide des formules élémentaires que nous venons d'indiquer, il est possible de traiter les cas les plus généraux de conduites ancrées entre deux massifs et formées de tronçons de métal et de caractéristiques différentes, avec on non, un obturateur (vanne ou robinet) intercalé.

La méthode la plus pratique, pour éviter les erreurs, est la suivante :

$\left.1^{\circ}\right)$ On calcule la flexibilité totale $K$ de toute la conduite; $k$ est la somme des flexibilités $k 1, k 2, k 3$, etc.. de chaque troncon donnée par la formule $k=\frac{l}{\mathrm{E} \Omega}$

On a intérèt à prendre $k$ en millimètres par tonne.

Avec $l$ en $\mathrm{mm} ; \Omega$ en $\mathrm{mm}^{2} \mathrm{E}$ en tonnes par $\mathrm{mm}^{2}$.

$$
\mathrm{E}=20 \text { tomnes } / \mathrm{mm}^{2} \text { pour l'acier. }
$$

$\left.2^{\circ}\right)$ On calcule le déplacement total de la conduite el les efforts longitudinaux en supposant que la conduite est à déplacement libre, c'est-à-dire qu'on suppose qu'il y a un joint coulissant à l'entrée du massif aval.

Les déplacements dus à la température sont : $B=b 1+b 2+b 3 \ldots \ldots \ldots+b n$

Les déplacements dus à la pression sont : $\mathrm{C}=c 1+c 2+c 3 \ldots \ldots+c n$

Le déplacement total est :

$$
\Delta=\mathrm{B}+\mathrm{C}
$$

La réaction de la conduite sur le massif aval sera :

$$
F_{1}=\frac{\Delta}{K}
$$

Il suffira d'ajouter algébriquement cette forec à celles qui existent en un point quelconque de la conduite supposée libre à l'aval, pour obtenir les efforts en ce point.

\section{EXEMPLE}

Soit une conduite formée de troncons différents en épaisseur ou en métal et obturée entre les deux massifs. 


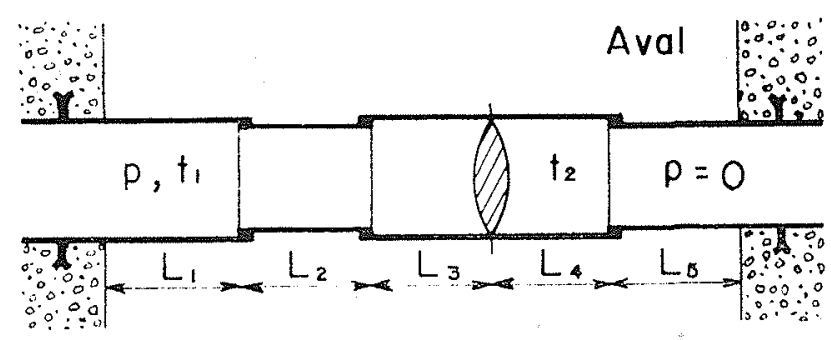

Fig. 6

LA PARTE GAUCHE DE I,OBTURATEUR EST EN PRESSION

$\left.1^{\circ}\right)$ On calcule la flexibilite :

$$
\mathrm{K}=k_{i}+k+k_{i ;}+k_{i}+k_{i ;}
$$

$2^{\circ}$ ) On suppose qu'il y a un joint coulissant à l'entrée du massif aval et on calcule les déplacements dus à la température. L'amont de lat conduite est sensiblement à la température de l'eau et l'aval à la température ambiante.

$b_{1}=\left(t_{1}-t_{0}\right) \propto l_{1}$ etc... $\quad b_{2}=\left(t_{1}-t_{0}\right) \alpha_{2} l_{2}$ etc... $b_{i}=\left(t_{2}-t_{0}\right) \alpha_{i} l_{i}$ etc... $\quad b_{;}=\left(t_{2}-l^{0}\right) \alpha_{i ;} l_{;}$etc... $\mathrm{B}=b+b_{2}+\ldots \ldots \ldots$

$3^{\circ}$ ) La partie aval est sans pression. Seule la partie amont est à la pression $p$ et, du fait qu'il y a obturateux, va s'allonger. On calcule les déplacements de chaque tronçon amont.

$$
\begin{aligned}
c_{1} & =\left(1 / 2-\eta_{1}\right) Q k_{1} \\
c_{2} & =\left(1 / 2-\eta_{2}\right) Q k_{2} \\
c_{3} & =\left(1 / 2-\eta_{3}\right) Q k_{3} \\
\mathrm{C} & =c_{1}+c_{2}+c_{3}
\end{aligned}
$$

$4^{\circ}$ ) Le déplacement total est $\Delta=\mathrm{B}+\mathrm{C}$. $5^{\circ}$ ) L'effort sur la conduite et le massif aval sera

$$
F_{1}=\frac{\Delta}{K}
$$

$\left.6^{\circ}\right)$ L'effort sur la conduite et le massif amont sera

$$
\mathrm{F}_{2}=\mathrm{Q}+\mathrm{F}_{1}
$$

Q et $F$ étant ajoulés algébriquement avec leurs signes respectifs.

\section{REMARQUES}

Les efforts sur les massifs ainsi trouvés sont unicuement les efforts dus aux réactions de la conduite située entre les deux massifs ; il est bien entendu qu'il y a lieu de rajouter les efforts transmis par les éléments de conduites situés de part et d'autre de ces massifs.

Il est nécessaire de traiter plusieurs cas poü voir quel est le plus défavorable.

Par exemple :

- conduite vide à différentes températures,

- conduite pleine avec obturateur ouvert,

- conduite avec eau en pression à l'amont $c^{2}$ eau sans pression à l'aval.

Il est aussi à remarquer que la flexibilité de la conduite peut être différente en traction et en compression. Par le fait, par exemple, de la flexibilité des brides d'assemblage, des joints en caoutchouc, etc...

On voit d'après les calculs précédents que la température de pose de la conduite a une grande importance, et il est indispensable de faire le dernier joint au moment de la journée où la température est la plus favorable. 